\title{
Analysis on the Anisotropic Tensile Properties of Plain Weave Fabrics
}

\author{
By Masako Niwa,* Sueo Kawabata** and Hiromichi Kawai** Members, TMSJ \\ *Department of Clothing Science, Nara Women's University, Nara \\ **Department of Polymer Chemistry, Kyoto University, Kyoto
}

Based on the Journal of the Textile Machinery Society of Japan, Transactions, Vol. 22, No. 11, T-256-269 (1969)

Abstract

The anisotropic tensile property of plain weave fabrics is theoretically analyzed as a development of the shear deformation theory and the biaxial deformation theory developed by the authors.

By this theory, the anisotropic properties of plain weave fabrics in the biaxial tensile field, including uniaxial tensile state, can be calculated. The conditions for this calculation are three mechanical properties of yarns, i.e., tensile, lateral compressive and frictional properties, and fabric structure. For uniaxial anisotropic property, some approximated methods are used in the theory.

When the principal tensile axis is rotated by $\Theta$ from the structure principal axis on the fabric plane, the tensile forces along the biased principal tensile axis are given by the following formulas for given $\lambda_{\text {and }} \lambda_{L}$, where $\lambda_{\text {and }} \lambda_{L}$ are stretch ratios along two principal tensile axes, respectively

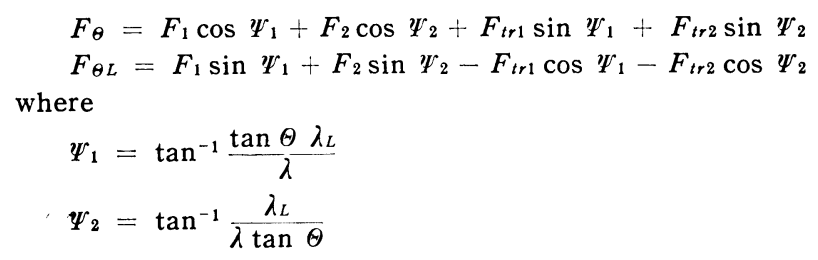

$F_{1}$ and $F_{2}$ are introduced as a function of $\lambda$ and $\lambda_{L}$ by the biaxial deformation theory. $F_{t r 1}$ and $F_{t r 2}$ are introduced as a function of $\lambda_{\text {and }} \lambda_{L}$ by the biaxial deformation theory and by yarn frictional property described in the shear deformation theory.

For uniaxial:

$$
\begin{aligned}
& F_{\theta}=\frac{F_{t r 1}}{\sin \Psi_{1}}+\frac{F_{t r 2}}{\sin \Psi_{2}} \\
& \text { and } \\
& \lambda=\frac{y_{01} \cos \Psi_{1}+y_{02} \cos \Psi_{2}}{y_{01} \cos \Theta+y_{02} \sin \Theta} \\
& \text { where } \\
& \begin{array}{l}
\Psi_{2}=\tan ^{-1}\left[\frac{y_{01}}{y_{02}} \frac{k_{2}}{k_{1}} \tan \Psi_{1}\right] \\
\phi=\frac{\pi}{2}-\left(\Psi_{1}+\Psi_{2}\right)
\end{array}
\end{aligned}
$$

$y_{01}$ and $y_{02}$ are yarn spacing for warp and weft respectively. $k_{1}$ and $k_{2}$ are constants and introduced from the biaxial deformation theory.

To calculate the relation between $F_{\theta}$ and $\lambda$, take $\Psi_{1}$ as a parameter. By varying the value of $\Psi_{1}$, the corresponding values of $F_{\theta}$ and $\lambda$ can be calculated. This means that the value of $F_{\theta}$ is a value corresponding to $\lambda$. Thus, the relation between $F_{\theta}$ and $\lambda$ can be obtained.

Experimental researches are also carried out for confirming theoretical calculation and good agreement between experimental and theoretical results is obtained.

KEY WORDS: FABrics, PLAIN WEAVES, ANISOTROPy (STRESS STRAIN), STRESS, TENSILE PROPERTIES, UNIAXIAL STRESS, BIAXIAL STRESS, SHEAR (MODE OF DEFORMATION), MECHANICAL PROPERTIES, THEORIES. 


\section{Introduction}

Fabrics have unique mechanical properties, in particular the tensile properties, unlike ordinary continuous materials.

The tensile properties of the fabrics when uniaxially stretched in either direction of warp or weft or biaxially in both directions at a time with a certain stretch ratio could be calculated with high accuracy. ${ }^{[1-4]}$

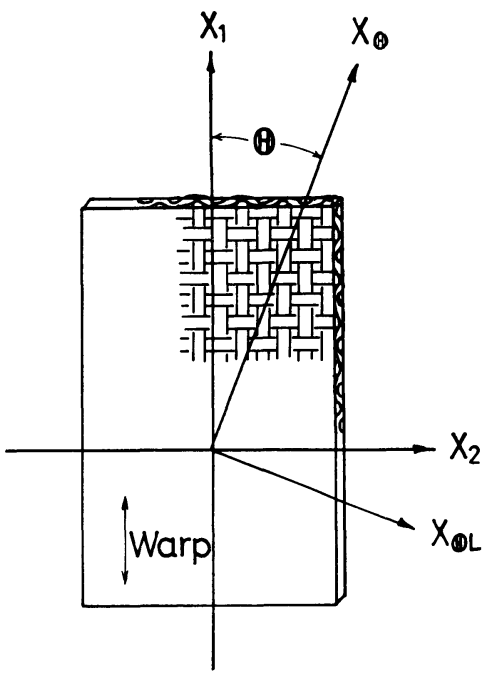

Fig. 1 Tho prinzipal axes of the structure of woven fabric are shown by $X_{1}$ and $X_{2}$, where $X_{1}$ axis is alonz warp and $X_{2}$ axis along weft. Axes $X_{\theta}$ and $\mathrm{X}_{\theta L}$ are taken along the biaxial tensile direstion and given by the rotation of the coordinate system of $\mathrm{X}_{i}$ by $\Theta$

The present paper, as illustrated in Fig.1, analyzes the bi- and uniaxial anisotropic tensile properties of plain weave fabrics along the newly drawn axis $X_{\theta}$ rotated by $\Theta$ from the structural principal axis $\mathrm{X}_{1}$.

The anisotorpy of the uni-axial tensile properties of plain weave fabric is measured at intervals of $15^{\circ}$ from the warp axis $X_{1}$, as shown in Fig.2, and the tensile force markedly varies according to direction. The tensile force required for the extension along the direction rotated by about $45^{\circ}$ from $X_{1}$ axis is approximately $10^{-1}$ to $10^{-2}$ times that of the structural-principal axis in same stretch ratio.

Such an extreme anisotorpy is presumably because of the low-resistance of the woven fabrics against shear force.

In $1949, \mathrm{~K}$. Weisse nberg et $\mathrm{a}^{[5,6]}$ published a paper on uni-axial tensile anisotropic properties of fabric. In their study, applying a method of simulating the fabric to a lattice model, they analyzed the fabric as a continuous material. Then, W.F.Kilby ${ }^{[7]}$ analyzed the tensile anisotropy of the fabric with the aid of this simple lattice model by regarding the fabric as a thin layer which has an anisotropy together with Poissons effect. Fukatani ${ }^{[8]}$ also used the same method in his study.

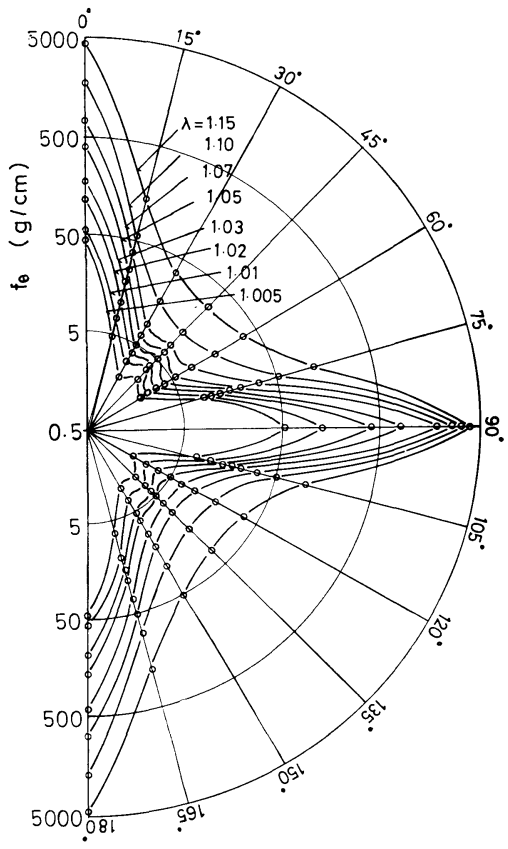

Fig. 2 Anisotropy of the uniaxial tensile property, measured sample is No. 3 fabric woven from polyester spun yarn shown in table 1

In their research, the tensi'e anisotropy was represented by the properties in warp, weft and $45^{\circ}$ directions. In other words, it is to treat the fabric as an orthotropic elastic material. However, the fabric is not a continuous material but discontinuous one.

It is doubted that such a method can ke used in discontinuous materials.

In this paper, the tensile anitostropic properties are analyzed by expanding the shear deformation theory of plain woven fabric developed by authors and are derived theoretically from the fabric structure and the mechanical properties of yarn used for the fabric.

On the other hand, the anisotropic properties in one or two dimensional-stress field are measured by the biaxial tensile tester ${ }^{[11]}$ deve'oped by the authors and the theoretical values are compared with the measured one.

\section{Theory}

2-1. Basic Structure Model

Let us consider an unit structure of plain woven fabric as enclosed by dotted line in Fig. 3. The unit structure includes a crossing point.

Fig. 4 shows coordinate systems before and after deformation.

$\mathrm{X}_{1}, \mathrm{X}_{2}$ axis are taken along structural-central lines of the fabric tefore deformation which are orthogonal to each other, and $x_{1}, x_{2}$ axes are along them after stretching along $X_{\Theta}$ and $X_{\theta L} . X_{1}$ and $X_{2}$ axes are not orthogonal in general. 


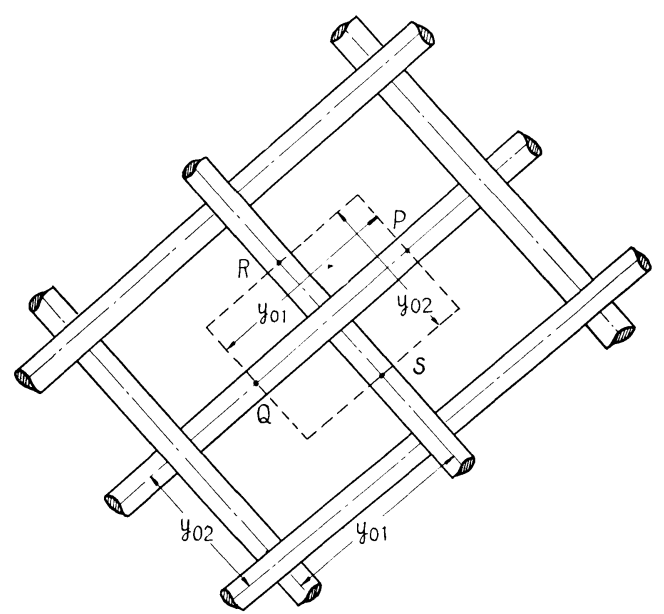

Fig. 3 An unit structure of plain woven fabric is shown by a datted line

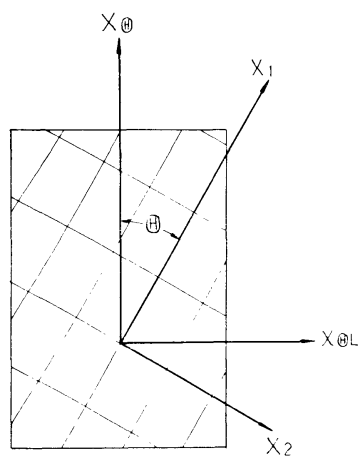

(a) undeformed state

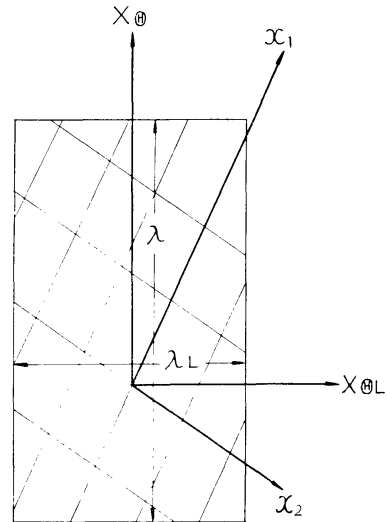

(b) deformed state
Fig. 4 Coordinate systems

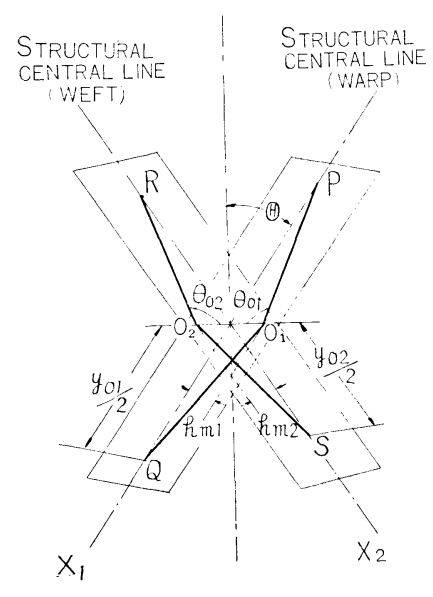

(a) undeformed state

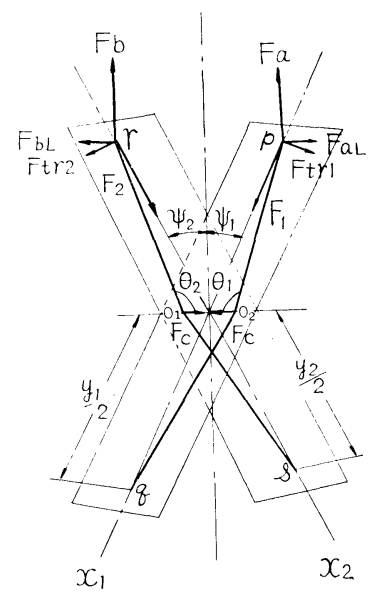

(b) deformed state
Fig. 5 Model of unit structure before and after deformation
As for the assumption and structural model, the biaxial tensile- and shear-deformation theories of the plain woven fabric already developed by authors can te applied.

The assumptions are:

(1) When the biaxial or uniaxial tensile deformation is given along the new $\mathrm{X}_{\theta}$ and $\mathrm{X}_{\theta L}$ axes, the structural central lines of the fabric do rot bend and keep straight lines.

(2) The weave angles of the weft and warp yarns do not te varied by change of the crossing angle. And the yarn tensions are dependent only on the stretch ratios along $\mathrm{x}_{1}$ and $\mathrm{x}_{2}$ axes and are not affected by the change of crossing angle.

Three dimensional representations of the unit model are shown in Fig. 5 where $P, Q, R$ and $S$ are correspondent to them in Fig.3.

Putting the warp density be $n_{1}$, weft density $n_{2}$, woven shrinkage ratio of warp and weft $S_{1}$ and $S_{2}$ respectively, tren the yarn spacings $y_{01}$ and $y_{02}$ and weave angles $\theta_{01}$ and $\theta_{02}$ in undeformed state are obtained by

$$
\begin{aligned}
& y_{01}=\frac{1}{n_{2}}, \quad y_{02}=\frac{1}{n_{1}} \ldots \ldots \ldots \ldots \ldots \ldots \ldots \ldots \ldots \ldots \ldots \ldots \ldots \\
& \theta_{01}=\sin ^{-1}\left(\frac{1}{S_{1}+1}\right), \quad \theta_{02}=\sin ^{-1}\left(\frac{1}{S_{2}+1}\right) \cdots \cdots \cdots(2)
\end{aligned}
$$

Then, the distances ketween the break points of yarn and the structure central line, $h_{m i}(i=1,2)$ can te obtained by

$$
h_{m 1}=\underset{2 \tan \theta_{01}^{-}}{y_{01}}, \quad h_{m 2}=\frac{y_{02}}{2 \tan \theta_{02}} \ldots \ldots \ldots \ldots \ldots
$$

\section{2-2. Anisotropy of the Biaxial Tensile Properties}

Let us consider the general deformation state shown in Fig.4(b) or Fig.5(b) where the biaxial tensile force is applied on the unit.

The projections of the unit model on $\mathrm{X}_{1}-\mathrm{X}_{2}$ plain or $\mathrm{x}_{1}-\mathrm{x}_{2}$ plain are shown in Fig.6, where the dotted lines and the solid lines present the undeformed and deformed state respectively. Now consider the general tensile deformation where the stretch ratio $\lambda$ is given along the axis $X_{\theta}$ and at the same time $\lambda_{L}$ along the axis $\mathrm{X}_{\theta L}$, vertical to theaxis $\mathrm{X}_{\theta}$. Here let the tensile forces in each direction te $\mathrm{F}_{\Theta}$ and $\mathrm{F}_{\theta L}$.

As seen in Fig.6, the points $P, Q, R$ and $S$ tefore the deformation move to the points $p, q, r$, and $s$ after the deformation. The external force $F_{\theta}$ can te divicicd into $F_{a}$ $F_{b}$ and the forces act at $p$ and $r$ respectively. And in same manner, $F_{\theta L}$ is also divided into $F_{a L}$ and $F_{b L}$. These forces are in equilibrium with the yarn tension $F_{i}(i=1,2)$ and forces $F_{\text {tri }}$ which arise from the torque required for the rotation of the yarn around a crossing point.

Now let the angles between $\mathrm{x}_{1}$ axeis and the tensile direction be $\psi_{1}$ as shown in Fig.6, and cefine $\phi$ such that the crossing angle of warp and weft is varied by $\phi$ from $\pi / 2$ due to the tensile deformation of the fabric. Then the relation among these ang!es, as is clear from Fig.6, is 


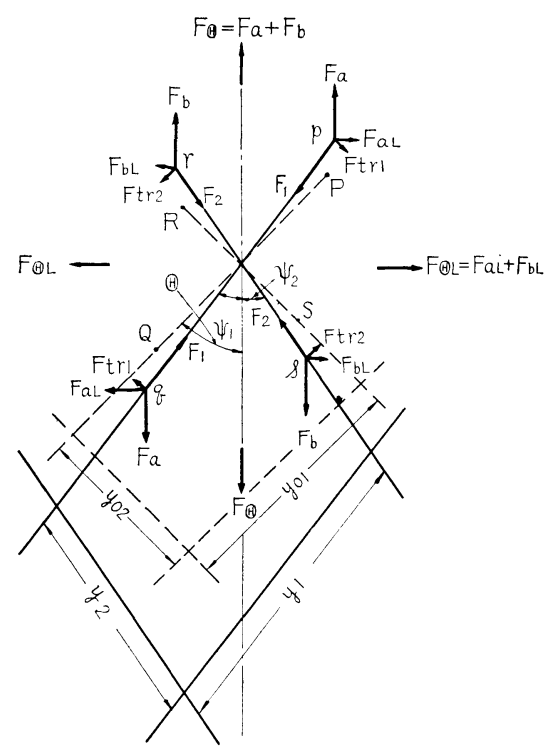

Fig. 6 Equilibrium of forces acting on an unit structure under biaxially stretched state

given as

$$
\begin{aligned}
& \Psi_{1}+\Psi_{2}=\frac{\pi}{2}-\phi \ldots \ldots \ldots \ldots \ldots \ldots(4) \\
& \phi=\phi_{1}+\phi_{2}, \quad \phi_{1}, \quad \phi_{2}>0 \cdots \cdots \cdots \cdots(5)
\end{aligned}
$$

The forces $F_{t r 1}$ and $F_{t r 2}$ are functions of $\phi$, as is clear from eqs. (16), (17) and (18).

From the equilibrium of the force, the following formule are obtained:

$$
\begin{aligned}
& F_{\theta}=F_{a}+F_{b} \\
& F_{\theta L}=F_{a L}+F_{b L} \ldots \ldots \ldots \ldots \ldots \ldots \ldots \ldots \ldots \ldots \ldots \ldots \\
& F_{a}=F_{1} \cos \Psi_{1}+F_{t r 1} \sin \Psi_{1} \\
& F_{b}=F_{2} \cos \Psi_{2}+F_{t r 2} \sin \Psi_{2} \\
& F_{a L}=F_{1} \sin \Psi_{1}-F_{t r 1} \cos \Psi_{1} \\
& F_{b L}=F_{2} \sin \Psi_{2}-F_{t r 2} \cos \Psi_{2} \\
& \frac{y_{1}}{2} F_{t r 1}=\frac{y_{2}}{2} F_{t r 2} \ldots \ldots \ldots \ldots \ldots
\end{aligned}
$$

From these equilibrium equations, $F_{\theta}$ and $F_{\theta L}$ under the biaxial stretching at given stretch ratios $\lambda_{\text {and }} \lambda_{L}$ can be calculated.

Substituting eqs.(8) and (9) into eq.(6),

$$
\begin{aligned}
F_{\theta}=F_{1} \cos \Psi_{1} & +F_{2} \cos \Psi_{2}+F_{t r 1} \sin \Psi_{1} \\
& +F_{t r 2} \sin \Psi_{2} \ldots \ldots \ldots \ldots \ldots \ldots \ldots(13)
\end{aligned}
$$

Also, substitute eqs.(10) and (11) into eq.(7), and

$$
\begin{aligned}
F_{6 L}=F_{1} \sin \Psi_{1} & +F_{2} \sin \Psi_{2}-F_{t r 1} \cos \Psi_{1} \\
& -F_{t r 2} \cos \Psi_{2} \ldots \ldots \ldots \ldots \ldots \ldots \ldots(14)
\end{aligned}
$$

In the case of $\lambda=\lambda_{L}, \Theta=0$, or $\Theta=\pi / 2$, we have

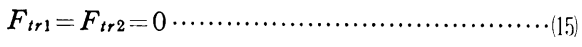

Otherwise

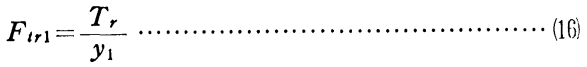

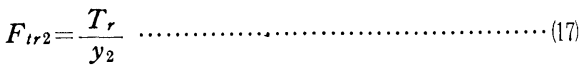

Here, $T_{r}$, when the crossing angle of the yarn is changing, will take the value approximated by eq.(18). Otherwise, i.e., when $\Theta$ is 0 or $\pi / 2$ and is stretched biaxially with equal stretch ratios, $F_{t r 1}$ and $F_{t r 2}$ are zero.

How the torque is affected by the degree of variation of crossing angle at the crossing point of warp and weft was already clarified in the shear-deformation theory. ${ }^{[9,10,12]}$ Namely, considering $T_{r}$ as a function of $F_{c}$ and $\phi$ where $F_{c}$ is the compressible force between warp and weft at their crossing point and $\phi$ is the rotated angle from the original rectangle at the point.

$$
T_{r}= \pm T_{r 0} \pm\left(C_{1}+C_{2} \phi\right) F_{c}+\left(C_{3}+C_{4} F_{c}\right) \phi \cdots \cdots(18)
$$

where $T_{r o}, C_{1}, C_{2}, C_{3}$ and $C_{4}$ are all constants and positive signs in the 1 st and 2 nd terms of the right of eq.(18) is used for the increasing process of $\phi$ and negative ones for the decreasing process of $\phi$. The 1 st and 2 nd terms represent the frictional component and the 3 rd terms elastic component.

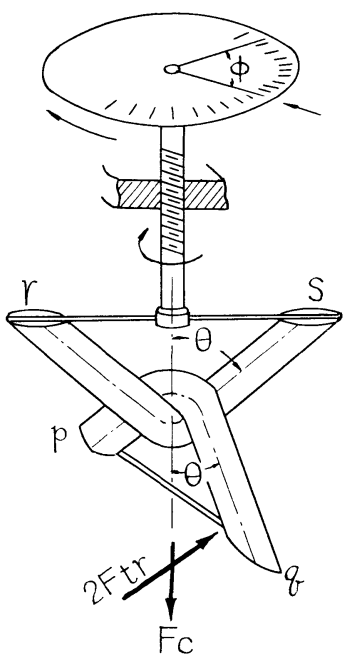

Fig. 7 Principle of the measurement of torque caused by rotation of crossing yarn at a crossing point and torque constants are derived from this measurement

The constants included in eq.(18), say torque constants, can be directly obtained by measuring the torque as shown in Fig. ${ }^{[12]}$ Those constants can be also derived from the shear-deformation properties of the fabric. ${ }^{[13]}$ Here the torque constants are treated as a characteristic value obtained by measurement of the yarn and the theoretical derivation of them is put out of our consideration.

On the other hand, $F_{1}, F_{2}$ and $F c$ are given as a function 
of $\lambda$ and $\lambda_{L}$ respectively for the calculation of $F_{\theta}$ and $F_{\theta L}$ by eqs.(13) and (14). In the first place the yarn spacings $y_{01}$ and $y_{02}$ become $y_{1}$ and $y_{2}$ respectively by biaxial stretching of stretch ratios $\lambda_{\text {and }} \lambda_{L}$.

$$
\begin{aligned}
& y_{1}=\sqrt{\left(y_{01} \sin \Theta \lambda_{L}\right)^{2}+\left(y_{01} \cos \Theta \lambda\right)^{2}} \cdots \cdots \cdots \cdots(19) \\
& y_{2}=\sqrt{\left(y_{02} \cos \Theta \lambda_{L}\right)^{2}+\left(y_{02} \sin \Theta \lambda\right)^{2}} \quad \cdots \cdots \cdots(20)
\end{aligned}
$$

Also, $\lambda_{1}$ and $\lambda_{2}$ which are the stretch ratios along structural-principal axes can be calculated by

$$
\begin{aligned}
& \lambda_{1}=\frac{y_{1}}{y_{01}}=\frac{\sqrt{\left(y_{01} \sin \Theta \lambda_{L}\right)^{2}+\left(y_{01} \cos \Theta \lambda\right)^{2}}}{y_{01}} \cdots(21) \\
& \lambda_{2}=\frac{y_{2}}{y_{02}}=\frac{\sqrt{\left(y_{02} \cos \Theta \lambda_{L}\right)^{2}+\left(y_{02} \sin \Theta \lambda\right)^{2}}}{y_{02}} \cdots \cdots(22)
\end{aligned}
$$

From the assumptions 1 and 2, the tensile stretch forces $F_{1}$ and $F_{2}$ and the compressive force $F c$ at the yarn-crossing point can be calculated as functions of $\lambda_{1}$ and $\lambda_{2}$, by applying the biaxial tensile deformation theory, by the following equations, ${ }^{[2-4]}$

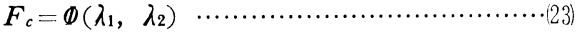

$$
\begin{aligned}
& F_{1}=g_{1}\left(\lambda_{1}, \quad \lambda_{2}\right)=\frac{F_{c} \lambda_{1} y_{01}}{4\left(h_{m 1}-h_{1}\right)} \cdots \cdots \cdots \cdots \cdots \cdots \cdots(24) \\
& F_{2}=g_{2}\left(\lambda_{1}, \lambda_{2}\right)=\frac{F_{c} \lambda_{2} y_{02}}{4\left(h_{m 2}+h_{2}\right)} \cdots \cdots \cdots \cdots \cdots \cdots(25)
\end{aligned}
$$

where $h$ is a function of $\lambda_{1}$ and $\lambda_{2}$ and defined by the following equation.

$$
h_{i}=K_{i}\left(\lambda_{1}, \lambda_{2}\right) \quad(i=1,2)
$$

The values of $h_{m 1}$ and $h_{m 2}$ are calculable from eq.(3), and $h_{i}$ is the displacement of point $o_{1}$ or $o_{2}$ in Fig.5 along $X_{3}$ axis, and can be calculated from tensile and compressive properties of the yarn and from fabric structure ${ }^{[3-4]}$ under any biaxial deformation.

$\Psi_{1}$ and $\Psi_{2}$ are calculated as following

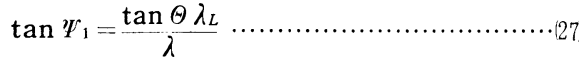

$$
\begin{aligned}
& \tan \Psi_{2}=\frac{\lambda_{L}}{\lambda \tan \Theta}
\end{aligned}
$$

Accordingly, the angle $\phi$ can be given by substituting eqs.(27) and (28) into eq.(4).

$$
\phi=\frac{\pi}{2}-\left\{\tan ^{-1}\left(\frac{\tan \Theta-\lambda_{L}}{\lambda}\right)+\tan ^{-1}\left(\frac{\lambda_{L}}{\lambda \tan \Theta}\right)\right\} \cdots(29)
$$

After calculating $F c$ by eq.(23) and $\phi$ by eq.(29), then $F_{t r 1}$ and $F_{t r 2}$ are obtainable as following. Substituting eqs.(8) and (19) into eq.(16) to obtain,

$$
F_{t r 1}=\frac{ \pm T_{r 0} \pm C_{1} F_{c}+\left(C_{3}+C_{4} F_{c} \pm C_{2} F_{c}\right) \phi}{\sqrt{\left(y_{01} \sin \Theta \lambda_{L}\right)^{2}+\left(y_{01} \cos \Theta \lambda\right)^{2}}} \cdots \cdots(30)
$$

And, substituting eqs.(18) and (20) into eq.(17),

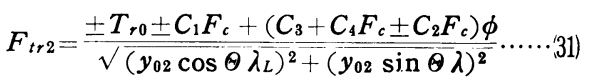

Finally $F_{\theta}$ and $F_{\theta L}$ are derived by eqs.(13) and (14), where $F c, F_{1}$ and $F_{2}$ can be calculated by the biaxial tensile deformation theory ${ }^{[3]}$ of the fabric. Here, note that $F_{\theta}$ and $F_{\theta L}$ are tensile loads per unit structure and the load per unit length, $f_{\Theta}$ and $f_{\theta L}$ correspondingly, are obtained as follows:

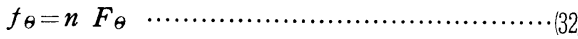

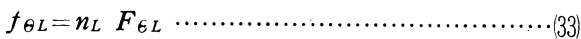

where,

$$
\begin{aligned}
n & =\frac{1}{y_{01} \sin \Theta+y_{02} \cos \Theta} \cdots \cdots \cdots \ldots \ldots \ldots \ldots \ldots(34) \\
n_{L} & =\frac{1}{y_{01} \cos \Theta+y_{02} \sin \Theta} \cdots \cdots \cdots \cdots \cdots \cdots \cdots \cdots \cdots(35)
\end{aligned}
$$

\section{2-3. Anisotorpy in Uniaxial Tensile Properties}

Uniaxial tensile deformation is a special case of the biaxial deformation where the force $F_{\theta L}$ is kept zero during the deformation.

This situation makes the analysis very difficult, so that some approximations are required. The equilibrium state of forces in unit structure is as shown in Fig.8. The external force $F_{\theta}$ consists of forces $F_{a}$ and $F_{b}$ acting on points $p$ and $q$ respectively. $F_{a L}$ and $F_{b L}$ are both disappeared because of uniaxial extension.
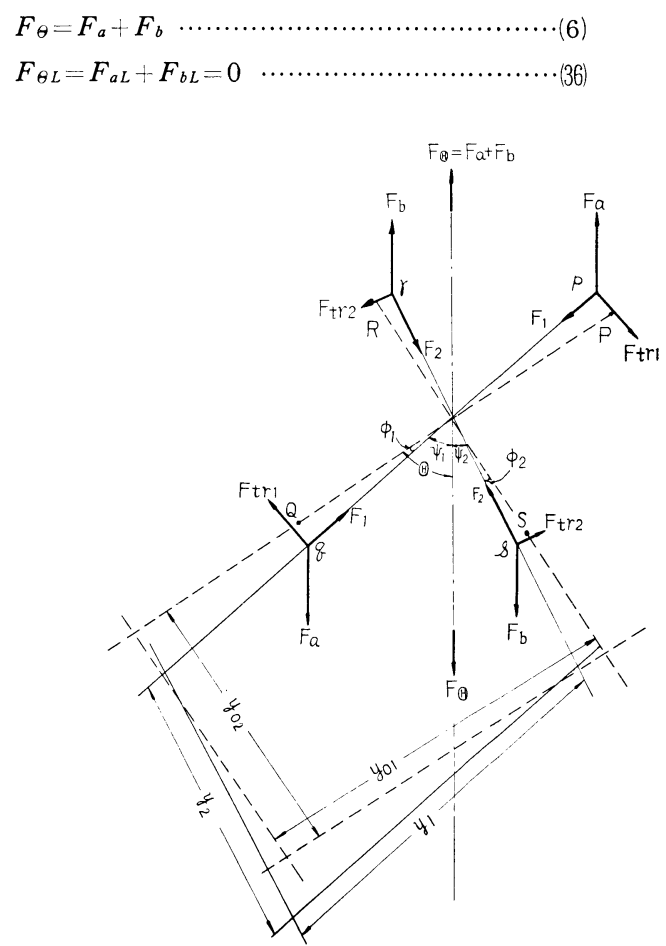

Fig. 8 Forces in equilibrium state of an unit structure under the uniaxially stretched state

The equilibrium equations (10) and (11) can be rewritten by using the relation of eq.(36), that is,

$$
\begin{aligned}
& F_{1} \sin \Psi_{1}-F_{t r 1} \cos \Psi_{1}=0 \\
& F_{2} \sin \Psi_{2}-F_{t r 2} \cos \Psi_{2}=0
\end{aligned}
$$


Accordingly, in the case of uniaxial deformation, the equilibrium equations presented by formule (6), (36), (8), (9), (37), (38) and (12) can be established.

$$
\begin{aligned}
F_{\theta}=F_{1} \cos \Psi_{1}+F_{2} \cos \Psi_{2} & +F_{t r 1} \sin \Psi_{1} \\
& +F_{t r 2} \sin \Psi_{2} \cdots \cdots(13)
\end{aligned}
$$

From eq.(37),

$$
F_{1}=\frac{F_{t r 1} \cos \Psi_{1}}{\sin \Psi_{1}}
$$

and from eq.(38),

$$
F_{2}=\frac{F_{t r 2} \cos \Psi_{2}}{\sin \Psi_{2}}
$$

It is noted that the fabric is deformed biaxially even though the bulk stretching is uniaxial.

By substituting eqs.(39) and (40) into eq.(13), $F_{\theta}$ can be derived as:

$$
F_{\theta}=\frac{F_{t r 1}}{\sin \Psi_{1}}+\frac{F_{t r 2}}{\sin \Psi_{2}}
$$

where $F_{t r 1}$ and $F_{t r 2}$ can be presented by eqs.(16) and (17), and $T_{r}$ can te approximated by eq.(18).

In general, woven fabrics is very extensible by small extension force toward the biased direction from warp or weft direction in the uniaxial extension. (Fig.2)

Accordingly, $F_{1}$ and $F_{2}$ are small and, thereby, the tension on the yarn is extremely small.

This fact implies that the biaxial deformations along warp and weft directions of the fabric caused by this uniaxial extension should te very small. In these small deformation region it has kecome c'ear that the relation between $F_{c}$ and $F_{1}$ or between $F_{c}$ and $F_{2}$ can be approximated by a linear relation ${ }^{[2-4]}$ such that,

$$
F_{c} \simeq k_{1} F_{1} \quad F_{c} \simeq k_{2} F_{2} \ldots \ldots \ldots \ldots \ldots \ldots \ldots \ldots \ldots(42)
$$

where $k_{1}$ and $k_{2}$ are both constants calculated by the biaxial tensile deformation theory ${ }^{[2-4]}$. But the valces of $k_{1}$ and $k_{2}$ are depend on the deformation mode of the biaxial stretching. This it is assumed that the deformation mode is very close to the homogeneous biaxial ceformation, that is, the case of $\lambda_{1}=\lambda_{2}$.

Substituting eq.(42) into eq.(18),

$$
\begin{aligned}
T_{r} & = \pm T_{r 0} \pm\left(C_{1}+C_{2} \phi \pm C_{4} \phi\right) k_{1} F_{1}+C_{3} \phi \\
& = \pm T_{r 0} \pm\left(C_{1}+C_{2} \phi \pm C_{4} \phi\right) k_{2} F_{2}+C_{3} \phi \cdots \cdots \cdots(43)
\end{aligned}
$$

And s:ıbstituting eq.(43) into eq.(16),

$$
F_{1}=\frac{F_{t r 1} y_{1} \mp T_{r 0}-C_{3} \phi}{ \pm\left(C_{1}+C_{2} \phi \pm C_{4} \phi\right) k_{1}}
$$

Also, s.ıbstituting eq.(43) into eq.(17),

$$
F_{2}=\frac{F_{t r 2} y_{2} \mp T_{r 0}-C_{3} \phi}{ \pm\left(C_{1}+C_{2} \phi \pm C_{4} \phi\right) k_{2}}
$$

From exs.(44) and (39),

$$
F_{t r 1}=\frac{ \pm T_{r 0}+C_{3} \phi}{\left.y_{1}-\frac{ \pm\left(C_{1}+C_{2} \phi \pm C_{4} \phi\right) k_{1}}{\tan \mathscr{F}_{1}} \ldots \ldots \ldots \ldots \ldots \ldots, 46\right)}
$$

From eqs.(45) and (40),

$$
F_{t r 2}=\frac{ \pm T_{r 0}+C_{3} \phi}{y_{2}-\frac{ \pm\left(C_{1}+C_{2} \phi \pm C_{4} \phi\right) k_{2}}{\tan \Psi_{2}} \ldots \ldots \ldots \ldots \ldots,(4)}
$$

By substituting eqs.(46) and (47) into the equilibrium eq.(12) and by rearranging the terms,

$$
\begin{aligned}
y_{2}- & \pm \frac{\left(C_{1}+C_{2} \phi \pm C_{4} \phi\right) k_{2}}{\tan \Psi_{2}} \\
& =\frac{y_{2}}{y_{1}}\left[y_{1}-\frac{ \pm\left(C_{1}+C_{2} \phi \pm C_{4} \phi\right) k_{1}}{\tan \mathscr{W}_{1}}\right] \cdots \cdots \cdots(48)
\end{aligned}
$$

From eq.(48), the following is obtained:

$$
\begin{aligned}
\tan \Psi_{2} & =\frac{y_{1}}{y_{2}} \frac{k_{2}}{k_{1}} \tan \Psi_{1} \\
\Psi_{2} & =\tan ^{-1}\left(\frac{y_{1}}{y_{2}} \frac{k_{2}}{k_{1}} \tan \Psi_{1}\right) \ldots \ldots \ldots \ldots \ldots \ldots(49)
\end{aligned}
$$

In the light of eq.(49), the relation between $\Psi_{1}$ and $\Psi_{2}$ during the uniaxial tensile deformation can be obtained. It is noticed that this relation between $\Psi_{1}$ and $\Psi_{2}$ is independent of $\Theta$. This implies that if the specimen is stretched along $X_{\theta}$ axis uniaxially there will take place the bending of the specimen in the plain so as to keep the relation of eq. (49) (refer to Fig.19).

Substituting eqs.(46) and (47) into eq.(41), and eqs.(48) and (49) into eq.(41), $F_{\Theta}$ is approximately as given

$$
\begin{aligned}
F_{\theta}= & \frac{ \pm T_{r 0}+C_{3} \phi}{y_{1}-\frac{ \pm\left(C_{1}+C_{2} \phi \pm C_{4} \phi\right) k_{1}}{\tan \Psi_{1}}} \\
& \left(\begin{array}{c}
1 \\
\sin \Psi_{1}
\end{array} \frac{y_{1}}{y_{2}} \sin \left\{\tan ^{-1}\left(\frac{y_{1}}{y_{2}} \frac{k_{2}}{k_{1}} \tan \Psi_{1}\right)\right\}\right) \cdots(50)
\end{aligned}
$$

The relation tetween $F_{\theta}$ and $\Psi_{1}$ can te obtained from eq.(50), and the re'ation tetween $\Psi_{1}$ and $\Psi_{2}$ is given by eq.(49), where

$$
y_{1}=y_{01} \lambda_{1}, \quad y_{2}=y_{02} \lambda_{2} \ldots \ldots \ldots \ldots \ldots \ldots \ldots(51)
$$

As shown previously, the fabric can te stretched easily in biased direction from its structural-principal axes by small amoint of force, so that the stretching of the yarn should be negligible during the ceformation. So that, assume

$$
\lambda_{1}=\lambda_{2} \simeq 1
$$

then e..(51) will te approximated as

$$
y_{1}=y_{01}, \quad y_{2} \approx y_{02} \ldots \ldots \ldots \ldots \ldots \ldots \ldots \ldots \ldots(53)
$$

By using the relations of eq.(53), eq.(50) tecomes very simp'e as

$$
\begin{aligned}
& F_{\theta}=\frac{ \pm T_{r 0}+C_{3} \phi}{y_{01}- \pm \frac{\left(C_{1}-C_{2} \phi \pm C_{4} \phi\right) k_{1}}{\tan \Psi_{1}}}
\end{aligned}
$$

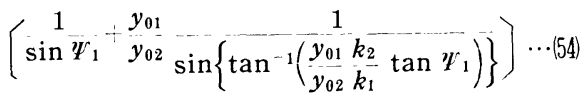

The load per unit spesinen width, $f_{\theta}$, can te calculated by the following equation where $n$ is the number of unit 
structure included in unit width of the fabric and given by eq.(34).

$$
f_{\theta}=n F_{\theta}
$$

On the other hand, the stretch ratio $\lambda$ when $\operatorname{load} F_{\theta}$ is applied can be geometrically calculated:

$$
\lambda=\frac{y_{1} \cos \Psi_{1}+y_{2} \cos \Psi_{2}}{y_{01} \cos \Theta+y_{02} \sin \Theta}
$$

Substituting eq.(49) into eq.(55) and applying eqs.(52) and (53),

$$
\lambda=\frac{y_{01} \cos \Psi_{1}+y_{02} \cos \left[\tan ^{-1}\left(\frac{y_{01}}{y_{02}} \frac{k_{2}}{k_{1}} \tan \Psi_{1}\right)\right]}{y_{01} \cos \Theta+y_{02} \sin \Theta} \ldots \ldots \ldots(56)
$$

The relati on between tensile load $F_{\theta}$ and stretch ratio $\lambda$ when the uniaxial tensile deformation is given toward the direction slant by a certain angle $\Theta$ from the structuralprincipal axes of the fabric can ce calculated by eqs.(54) and (56) incorporating the approximated values of eqs.(52) and (56).

Taking into consideration that both eqs.(54) and (56) include $\Psi_{1}$, the actual calculation can be made by such method, taking $\Psi_{1}$ as dummy parameter and giving this some dummy value, $F_{\theta}$ and $\lambda$ can be calculated by eqs.(54) and (56) respectively then $\lambda$ corresponding to the $F_{\theta}$ is obtained. This procedure is repeated for different value of $\Psi_{1}$.

\section{Comparison of Theoretical Values and Experimental Values}

To be sure of the accuracy of the theory, let us compare the theoretical and experimental values in the two cases: (1) the anisotropy of strip biaxial tensile deformation $\left(\lambda_{L}=1\right)$ and (2) the anisotropy of uniaxial tensile cieformation.

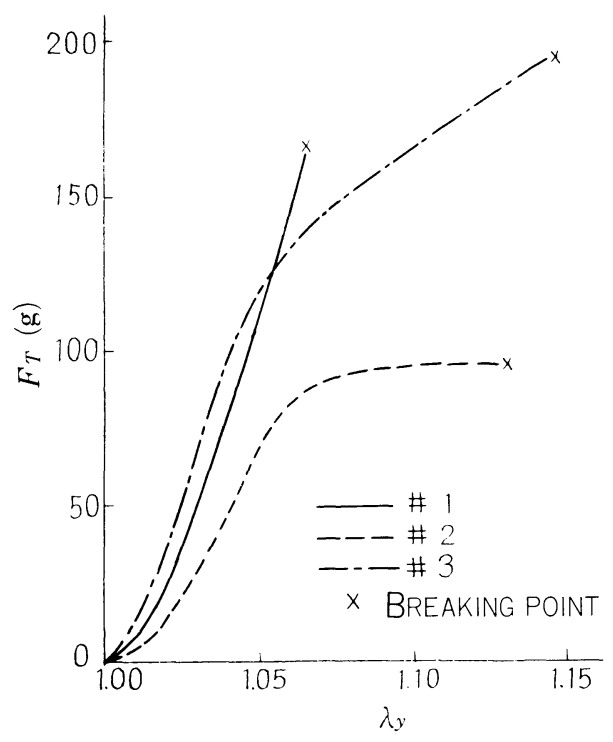

(a) tensile properties of samples No. 1 , No. 2 and No. 3 in table 1
Table 1. Structure constants of the plain woven fabric used for the experiment and calculation

\begin{tabular}{l|c|c|c|c|c|c|c}
\multicolumn{1}{|c|}{ Fibre } & \multicolumn{2}{|c|}{$\begin{array}{c}\text { Yarn } \\
\text { Density } \\
\left(\mathrm{cm}^{-1}\right)\end{array}$} & \multicolumn{2}{|c|}{ Yarn Crimp } & \multicolumn{2}{|c|}{$\begin{array}{c}\text { Yarn } \\
\text { Spacing } \\
\left(\times 10^{-2} \mathrm{~cm}\right)\end{array}$} \\
& $n_{1}$ & $n_{2}$ & $S_{1}$ & $S_{2}$ & $y_{01}$ & $y_{02}$ \\
\hline \$1 Cotton & 35.3 & 22.7 & 0.170 & 0.038 & 4.405 & 2.833 \\
\$2 Wool & 34.1 & 22.5 & 0.118 & 0.036 & 4.444 & 2.933 \\
\$3 Polyester & 34.9 & 21.0 & 0.130 & 0.018 & 4.762 & 2.865 \\
\hline
\end{tabular}

Table 2 Constants used in the calculation. Values of $k_{1}$

\begin{tabular}{|c|c|c|c|c|c|c|}
\hline \multirow{2}{*}{ Fibre } & \multirow{2}{*}{$k_{1}$} & \multirow{2}{*}{$k_{2}$} & \multicolumn{4}{|c|}{$\begin{array}{l}\text { Torque Constants } \\
\left(\times 10^{-3}\right)\end{array}$} \\
\hline & & & $\begin{array}{c}T_{r 0} \\
\text { g.cm) }\end{array}$ & $\begin{array}{c}C_{1} \\
(\mathrm{~cm})\end{array}$ & $\mid \begin{array}{c}C_{3} \\
(\mathrm{~g} . \mathrm{cm})\end{array}$ & $\begin{array}{c}C_{4} \\
(\mathrm{~cm})\end{array}$ \\
\hline$\$ 1$ Cotton & 1.09 & 0.48 & 2.22 & 2.65 & 0.45 & 0.67 \\
\hline$\$ 2$ Wool & 0.84 & 0.28 & 0.85 & 1.85 & 0.22 & 0.44 \\
\hline $\begin{array}{l}3 \\
\text { Polyester }\end{array}$ & 0.99 & 0.29 & 2.85 & 3.50 & 0.41 & 0.59 \\
\hline
\end{tabular}
and $k_{2}$ is calculated from the property of homogeneous-biaxial deformation of the fabric

The fabrics used for the experinent have such structural constants as shown in Table 1. Those thrce kinds of specimens are woven from 30's spun yarn and their structures are very close each other.

The tensile and compressible properties of these yarns are measured and shown in Fig.9. The torque constants to ke used for calculation is obtained by the method shown in Fig. 7 and are shown in Table 2.

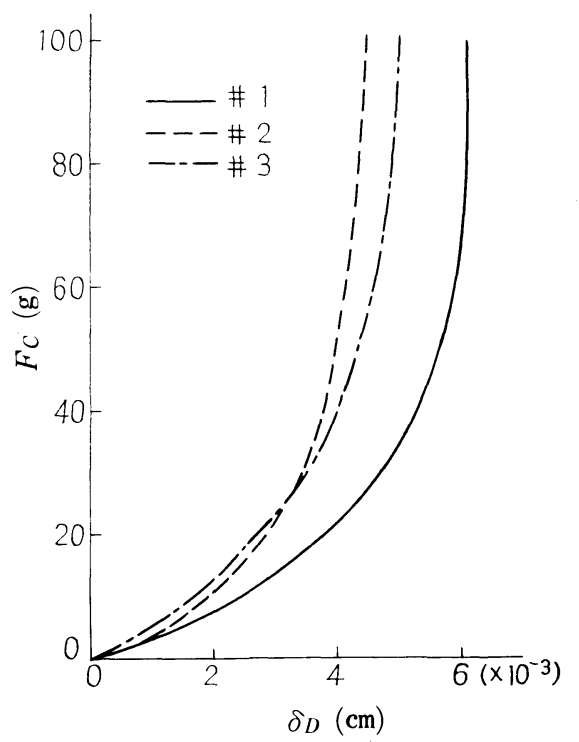

(b) compressible properties, $\delta_{D}$ is the amount of the compressive deformation of yarn

Fig. 9 Yarn properties used for theoretical calculation 


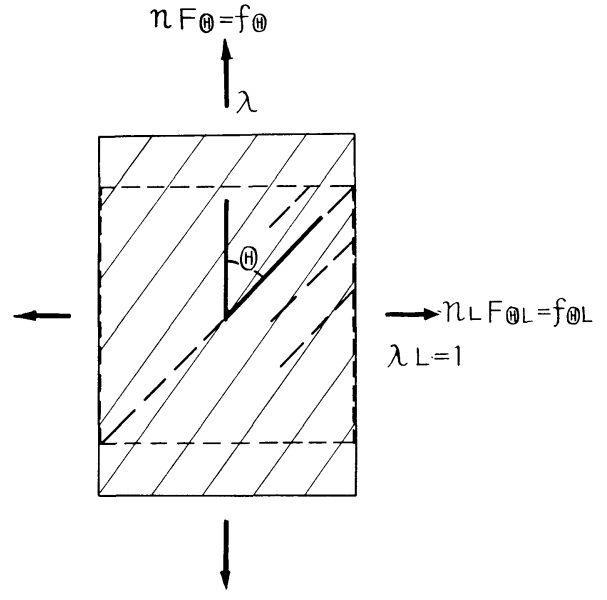

Fig. 10 Strip-biaxial extension along $X_{\theta}$ axis

The constants $k_{1}$ and $k_{2}$ are calculated by the biaxial deformation theory and also shown in Table 2.

3-1. Anisotropy of Biaxial Tensile Properties

Calculation of the tensile load $F_{\Theta}$ and constraint load $F_{\theta L}$ is done at first under the given condition that the fabric is stretched at tensile ratio $\lambda$ toward the axis $X_{\Theta} \mathrm{kept}$ $\lambda_{L}=1$ as shown in Fig. 10 .

From the given condition,

$$
\lambda_{L}=1
$$

\section{(57)}

Substituting eqs.(59) into (21) and (22), obtained

$$
\begin{aligned}
& \lambda_{1}=\frac{y_{1}}{y_{01}}=\frac{\sqrt{\left(y_{01} \sin \Theta\right)^{2}+\left(y_{01} \cos \Theta \lambda\right)^{2}}}{y_{01}} \ldots \ldots(58) \\
& \lambda_{2}=\frac{y_{2}}{y_{02}}=\frac{\sqrt{\left(y_{02} \cos \Theta\right)^{2}+\left(y_{02} \sin \Theta \lambda\right)^{2}}}{y_{02}} \ldots \ldots(59)
\end{aligned}
$$

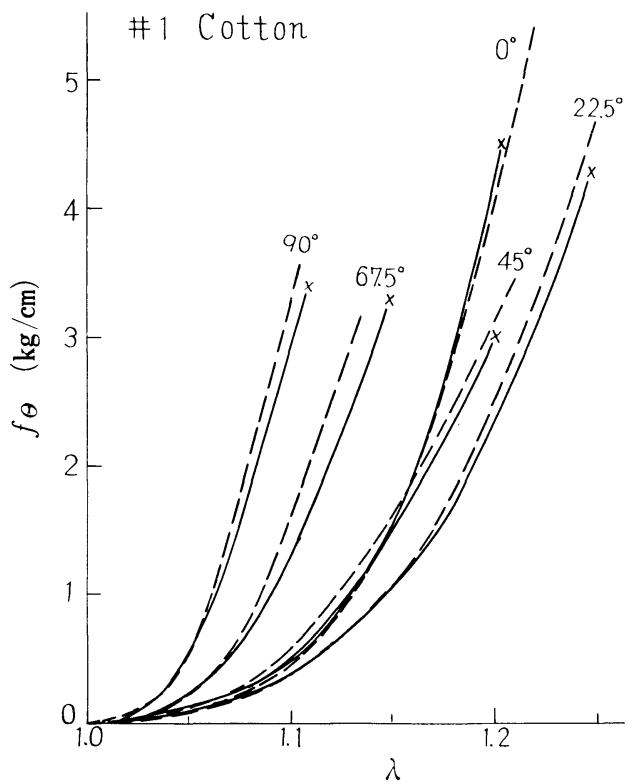

Fig. 11 Anisotropy in the tensile propertices of No. 1 cotton fabric under strip-biaxial extension
The tensile ratios $\lambda_{1}$ and $\lambda_{2}$ of the fabric along the axes $x_{1}$ and $x_{2}$ at tensile ratio $\lambda$ along $X_{\Theta}$ axes are calculable from the yarn spacings $y_{01}, y_{02}$ and biazed angle $\Theta$ by eqs.(58) and (59) respectively. As a particular case, if $\Theta=45^{\circ}$

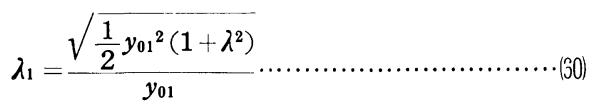

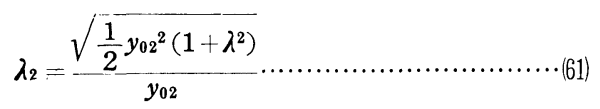

$F_{c}, F_{1}$ and $F_{2}$ can be calculated by the biaxial tensile deformation theory, ${ }^{[1-4]}$ using the structural constants of the fabric shown in Table 1 and the mechanical properties of the yarns shown in Fig.9. (For the details of the culculating method, refer to $\left.{ }^{[4]}\right)$.

Then, substituting eq.(57) into eqs.(27) and (28),

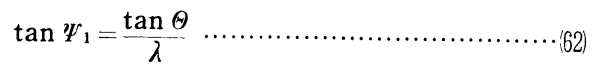

$$
\begin{aligned}
& \left.\tan \Psi_{2}^{\prime}=\frac{1}{\lambda \tan \Theta} \cdots \cdots \ldots \ldots \ldots \ldots \ldots \ldots \ldots \ldots \ldots \ldots \ldots, 63\right)
\end{aligned}
$$

In particular, when $\Theta=45^{\circ}$,

$$
\tan \Psi_{1}=\frac{1}{\lambda}, \quad \tan \Psi_{2}=\frac{1}{\lambda} \ldots \ldots \ldots \ldots \ldots \ldots \ldots(64)
$$

i.e.,

$$
\Psi_{1}=\Psi_{2}
$$

Angle $\dot{\rho}$ is obtained by substituting eqs.(62) and (63) into eq.(4)

$$
\left.\phi=\frac{\pi}{2}-\left\{\tan ^{-1}\left(\frac{\tan \Theta}{\lambda}\right)+\tan ^{-1}\left(\frac{1}{\lambda \tan \Theta}\right)\right\} \cdots \cdots \cdot 66\right)
$$

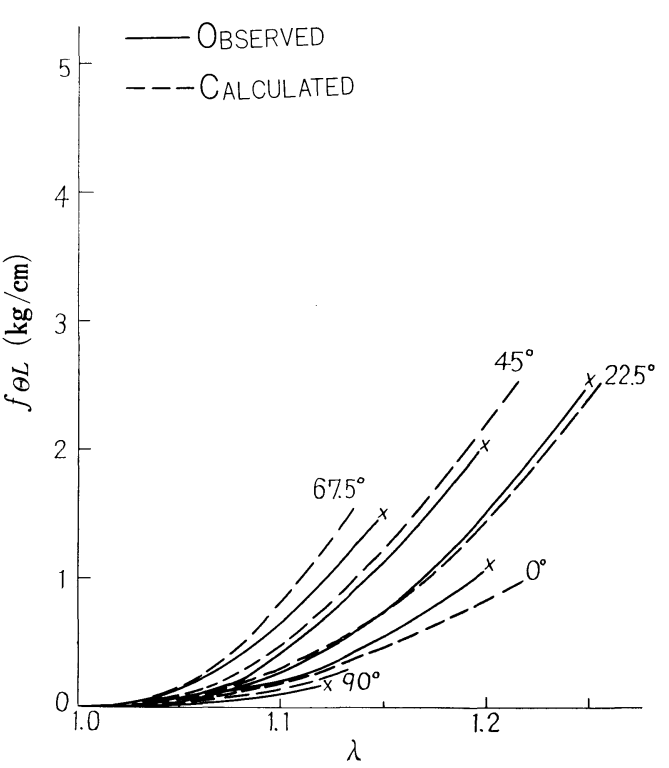



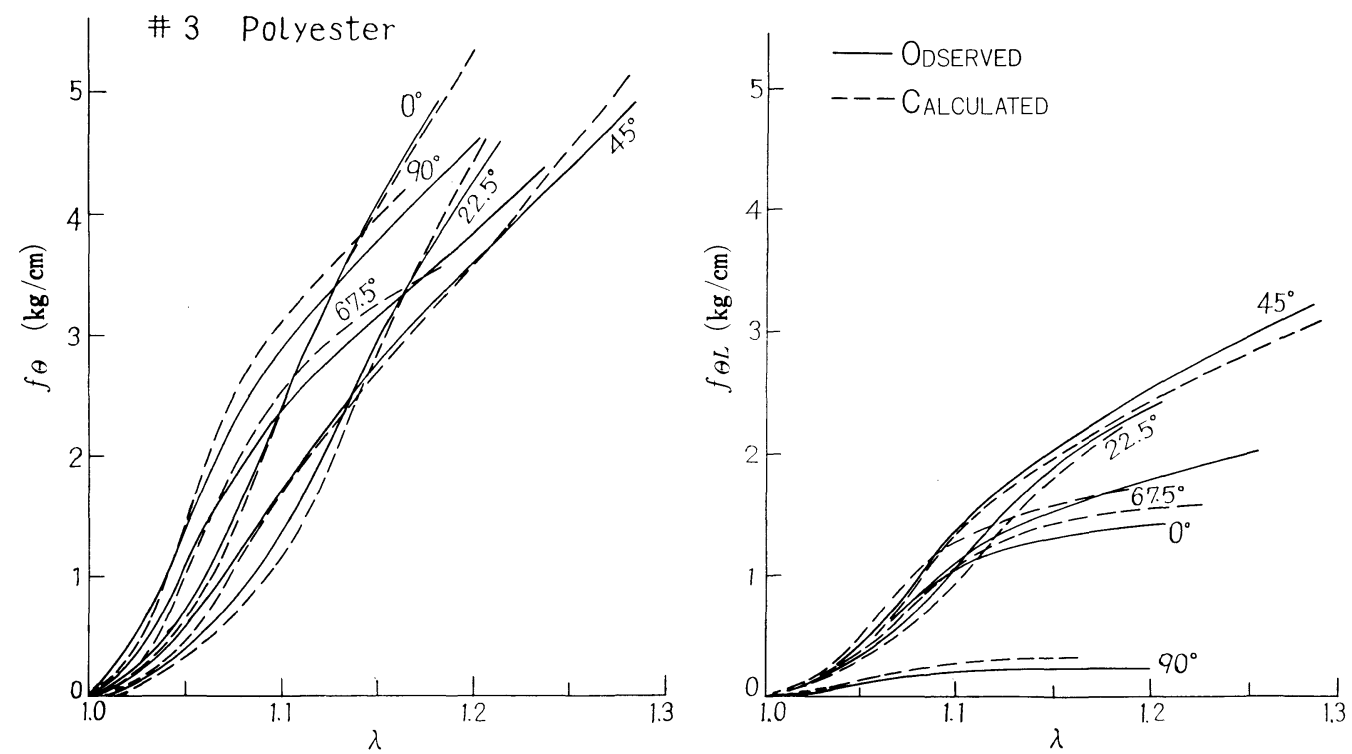

Fig. 12 Anisotropy in the tensile properties of No.2 wool fabric in strip-biaxial extension
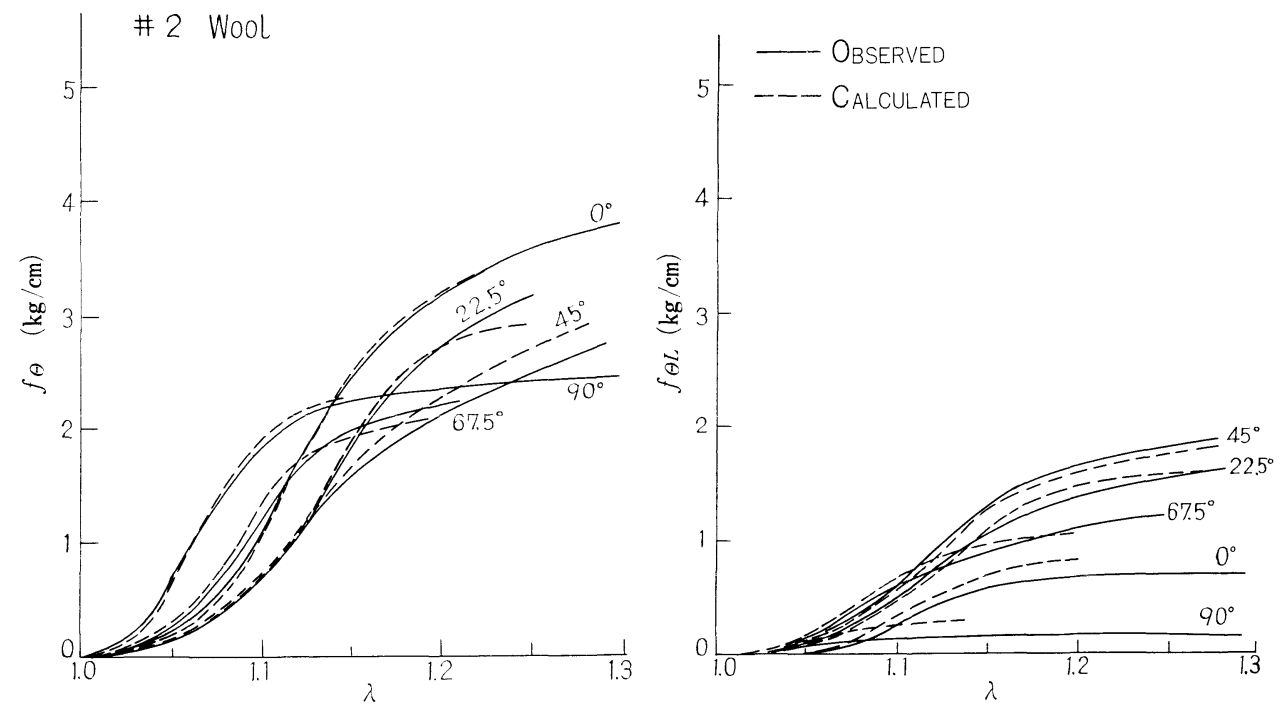

Fig. 13 Anisotropy in the tensile properties of No.3 fabric woven from polyester spun yarn in strip-biaxial extension

and when $\Theta=45^{\circ}$, substituting eq.(64) into eq.(4),

$$
\phi=\frac{\pi}{2}-2\left(\tan ^{-1} \frac{1}{\lambda}\right)
$$

Thus, $F c$ and $\phi$ can be calculated. Using the torque constants $T_{r o}, C_{1}, C_{3}$ and $C_{4}$ (in this case, $C_{2}=0$ ), $F_{t r 1}$ and $F_{t r 2}$ are calculable from eqs.(30) and (31) by incorporating eqs.(58) and (59), that is,

$$
\begin{aligned}
& F_{t r 1}=\frac{ \pm T_{r 0} \pm C_{1} F_{c}+\left(C_{3}+C_{4} F_{c}\right)}{\lambda_{1} y_{01}} \phi_{\ldots \ldots \ldots \ldots(68)} \\
& F_{t r 2}=\frac{ \pm T_{r 0} \pm C_{1} F_{c}+\left(C_{3}+C_{4} F_{c}\right) \phi \ldots \ldots \ldots \ldots(69)}{\lambda_{2} y_{02}}
\end{aligned}
$$

Substituting $F_{t r 1}$ and $F_{t r 2}$ calculated above into eqs. (13) and (14), and putting $\lambda_{L}=1, F_{\theta}$ and $F_{\theta L}$ are calculable.

If $n$ and $n_{L}$ are calculated by eqs.(34) and (35), then the load $f_{\Theta}$ per unit width and constraint load $f_{\Theta L}$ can be obtained. The results of this calculations for $\Theta=22.5^{\circ}, 45^{\circ}$ and $67.5^{\circ}$ are shown in Figs. 11 to 13 by dotted lines. On the other hand, the measurement of these properties have been carried out using the biaxaial tensile tester ${ }^{[11]}$ where the tensile velocity is selected as $50 \% / \mathrm{min}$. And the results are shown in same figures by solid lines for comparison. In these figures the tensile properties in the structural 


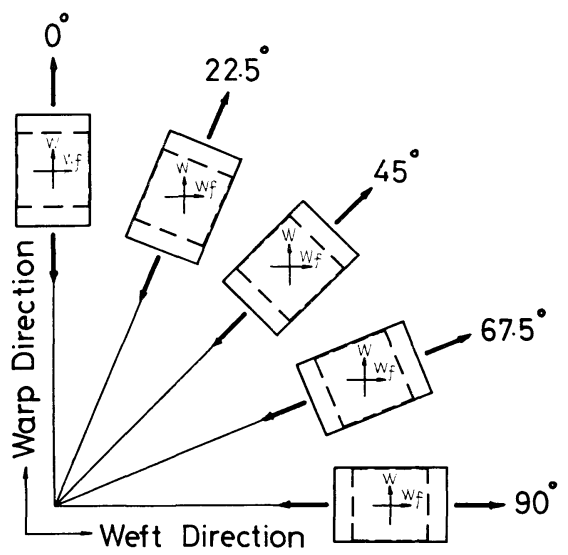

Fig. 14 Deformation modes

principal axes $X_{1}$ and $X_{2}$, i.e., in the case of $\Theta=0^{\circ}, 90^{\circ}$ are shown in the same figure.

As shown in Fig.14, warp direction of the specimens is taken as $\Theta=0^{\circ}$, arrow mark indicates the tensile direction.

Fig.15 is the photographic view. A good agreement tetween theoretical and experimental values is obtained all over the full region from small stretching to large stretching.

Samples shown in Figs. 12 and 13 haveyielding property and yet well-agreement between theoretical and measured values is observed after yielding. In Fig.16, these results are shown on polar coordinate as contour line of $\lambda$.

3-2. Anisotropy of Uniaxial Tensile Properties

Taking the approximation of $\lambda_{1}=\lambda_{2} \simeq 1$, i.e.,

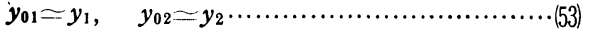

The anisotropy of the uniaxial stretching property is calculated by eqs.(54) and (56).

Giving $\psi_{1}$ as a parameter and substituting the approximated ralation of eq.(53) into eq.(49), $\Psi_{2}$ is calculated by

$$
\Psi_{2}=\tan ^{-1}\left(\frac{y_{01}}{y_{02}} \frac{k_{2}}{k_{1}} \tan \Psi_{1}\right)
$$

and $\phi$ is calculated by

$$
\phi=\frac{\pi}{2}-\left(\Psi_{1}+\Psi_{2}\right)
$$

The relationship ketween $\Psi_{1}, \Psi_{2}$ and $\phi$ can te decided regardless of $\Theta$, and the upper and the lower limits of $\Psi_{1}$ and $\Psi_{2}$ can te decided by constants $k_{1}$ and $k_{2}$ obtainable from fabric structures $y_{01}$ and $y_{02}$ and homogeneous

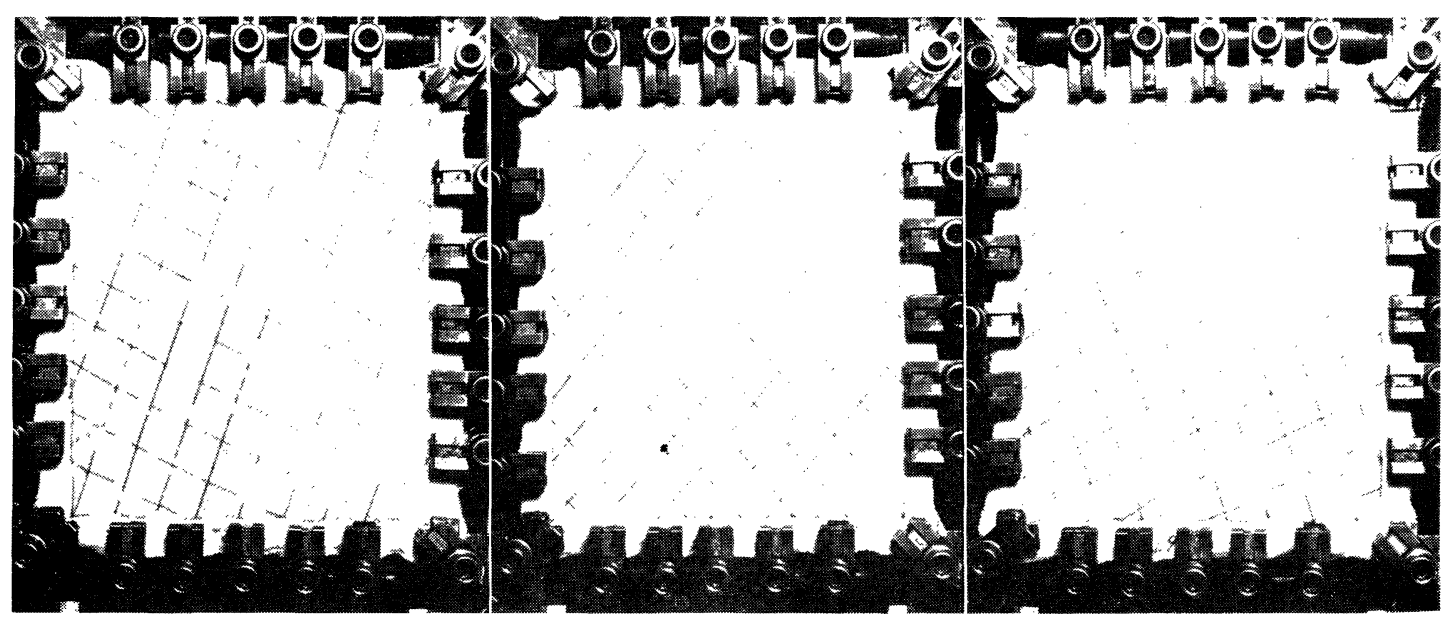

(a) $\Theta=22.5^{\circ}$

(b) $\Theta=45$

(c) $\Theta=67.5$

Fig. 15 The appearance of the samples under sing of the strip biaxial extension $\left(\lambda_{L}=1, \lambda=1.2\right)$
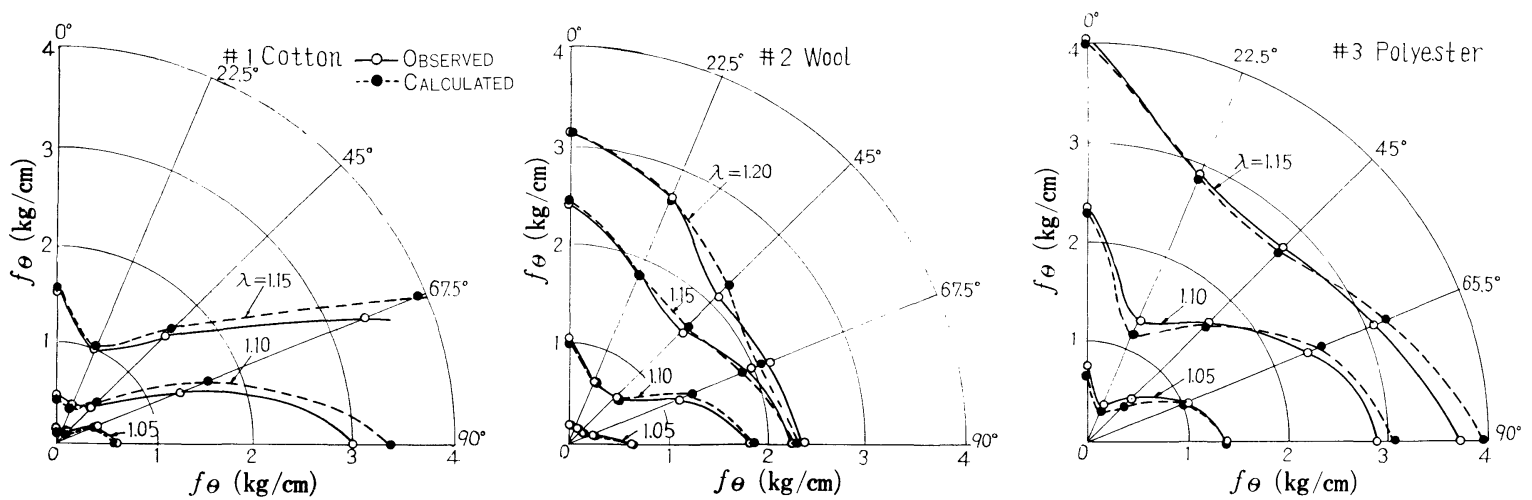

Fig. 16 Anisotropy in the tensile properties of No. 1, No. 2 and No. 3 samples in strip-biaxial extension 
biaxial tensile properties.

$F_{\theta}$ can be calculated substituting the torque constants and the value of $k_{1}$, which are shown in Table 2, into eq.(71) for any given parameter $\Psi_{1}$.

$$
F_{\theta}=\frac{ \pm T r_{0}+C_{3} \phi}{y_{01}-\frac{ \pm\left(C_{1} \pm C_{4} \phi\right) k_{1}}{\tan \Psi_{1}}}\left[\frac{1}{\sin \Psi_{1}}+\frac{y_{01}}{y_{02}} \frac{1}{\sin \Psi_{2}}\right] \cdots(71)
$$

$\lambda$ can be also calculated by eq.(72) for any given parameter $\Psi_{1}$.

$$
\lambda=\frac{y_{01} \cos \Psi_{1}+y_{02} \cos \Psi_{2}}{y_{01}}
$$$$
y_{01} \cos \theta+y_{02} \sin \theta
$$

In this way many sets of $\lambda$ and $F_{\theta}$ corresponding to many values of $\Psi_{1}$ are obtained. From this, the relation between $\lambda$ and $F_{\theta}$ is decided. Besides, the load $f_{\theta}$ per unit width can be calculated by $n F_{\theta}$.

However, in this case, $n$ is

$$
n=\frac{1}{y_{01} \sin \Theta+y_{02} \cos \theta}
$$

Accordingly, $f_{\theta}$ can be decided according to the angle $\Theta$.

Using constants of the samples (Tables 1 and 2), the calculation curve when the fabric stretched in the direction is $\Theta=45^{\circ}$ is shown with dotted lines in Fig. 17.

Experimental conditions used here was the following: specimen size is $2 \times 20 \mathrm{~cm}$ cut at intervals of $15^{\circ}$ or $22.5^{\circ}$, taking the warp direction as $0^{\circ}$; width to length ratio of sample, $1: 10$, and tensile speed is $50 \% / \mathrm{min}$. The solid lines of Fig. 17 show the experimental results. In this case, there is disagreement betwcen experimental and theoretical values in re'atively large-stretched region in which the $f_{\theta}-\lambda$ curve takes a steep curve. The reason is presumably that the yarn stretching can not ke neglected. Accordingly, as far as the approximation of eq.(53) is concerned, i.e., in the s.nall deformation region, the both curves are well agreed.

There is also a stepwise rise-up in the beginning of the deformation as seen is the shear deformation properties. This is a usual phenomenon seen in the uniaxial tensile properties in biased direction from warp or weft. This can be also theoretically calculated. The difference in the initial tensile properties ketween the specimens of Fig.17, comes from the difference of the torque constants of those specimen.

In the similar comparison, Fig. 18 shows the case of $45^{\circ}$. In this case, in particular, hysteresis calculation was also carried out. The material is the polyester spun yarn fabric.

As previously mentioned, for the recovery process of theoretical calculation is used the negative symbol of - in eq.(71).

As is clear from Fig.19, when the fabric is stretched toward the direction of $45^{\circ}$, both $\Psi_{1}$ and $\Psi_{2}$ take almost same values. The fabric is stretched evenly toward either direction of warp and weft. Recall the approximation that $k_{1}$ and $k_{2}$ are used for the value in the case of homogeneous

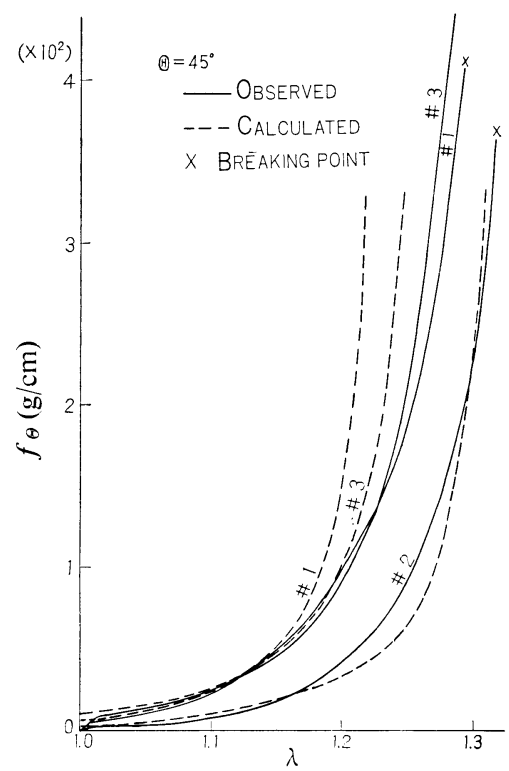

Fig. 17 Uniaxial tensile properties along $X_{\theta}$ axis. $\left(\Theta=45^{\circ}\right)$

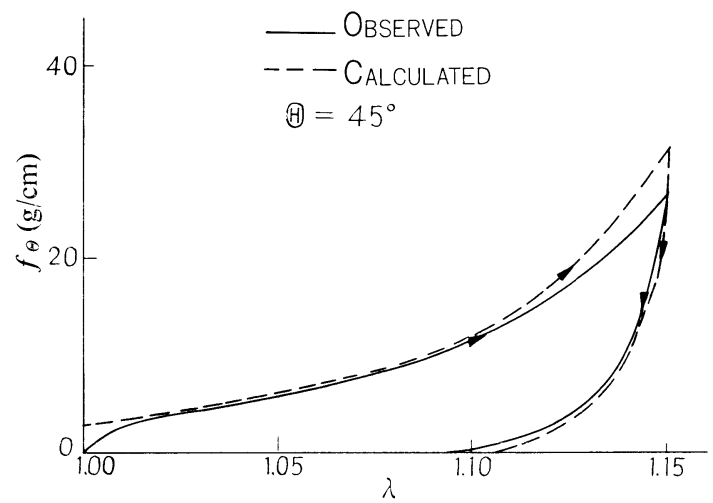

Fig. 18 Hysteresis in the uniaxial tensile property of fabric (polyester No.3 spun) along $X_{\theta}\left(\Theta=45^{\circ}\right)$ axis

biaxial stretching. Relatively highly accurate approximation is estimated around $45^{\circ}$ direction. In Fig. 19 , when $\Theta=45^{\circ}$, the fabric is stretched straight. But when $\Theta$ takes the other angle, there occurs some tending of specimen as shown in the figure which was expected by formula (70).

The pure uniaxial experiment is expected to te difficult in practice except in the case of $45^{\circ}$.

\section{Discussion}

4-1. Accuracy of the Approximated Solution for Anisotropic Property of the Uniaxial Tensile Deformation

Values of $k_{1}$ and $k_{2}$ in eq.(49) are measured to te equal to those obtained from the homogeneous-biaxial extension of the fabric toward the axes $x_{1}$ and $x_{2}$.

However, as is clear from the photographical observation, this assumption is reasonable for the tensile defor- 


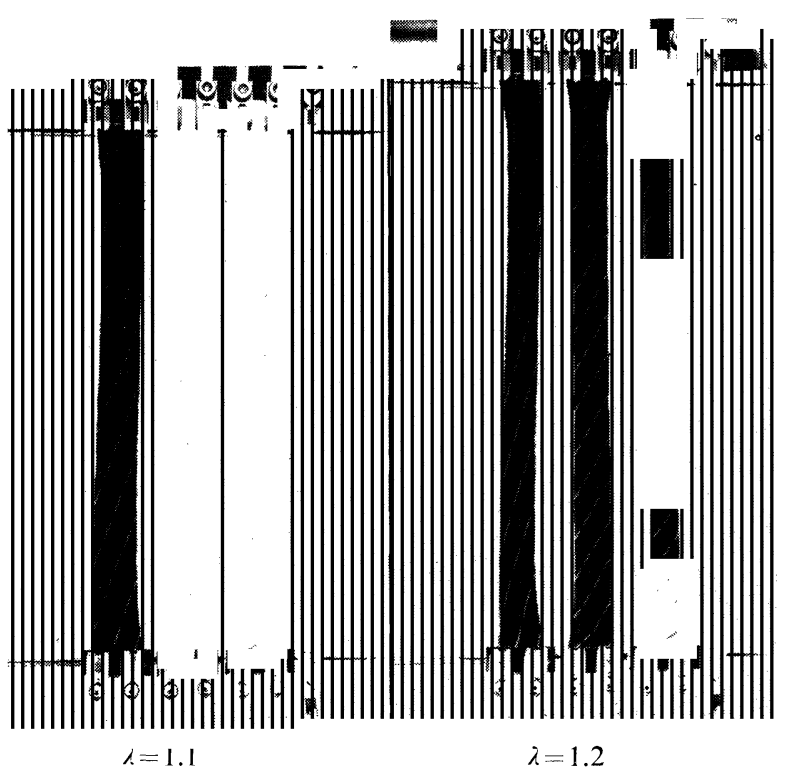

Fig. 19 The appearance of the samples under testing of uniaxial extension

(left $\Theta=22.5^{\circ}$, center $\Theta=45^{\circ}$, right $\Theta=67.5^{\circ}$ in each figure)

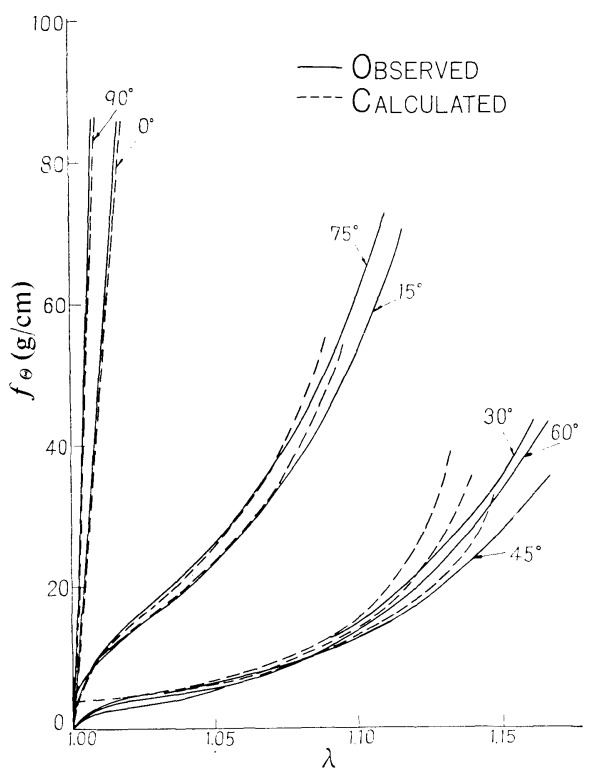

Fig. 20 Anisotropy in the tensile properties of No. 3 sample in uniaxial extension

$\left(\Theta=15^{\circ}, 75^{\circ}\right.$ are corrected using the corrected theory)

mation in the visinity of $\Theta=45^{\circ}$. But as it moves from $\Theta=45^{\circ}$ to $0^{\circ}$, the tensile ratio along the axis $x_{1}$ is greater than that of $x_{2}$ axis, and to $90^{\circ}, x_{2}$ is greater than $x_{1}$.

Accordingly, when $\Theta$ is $0^{\circ}$ or $90^{\circ}$, it seems to be a better approximation to use $k_{1}$ and $k_{2}$ obtained by strip-biaxial

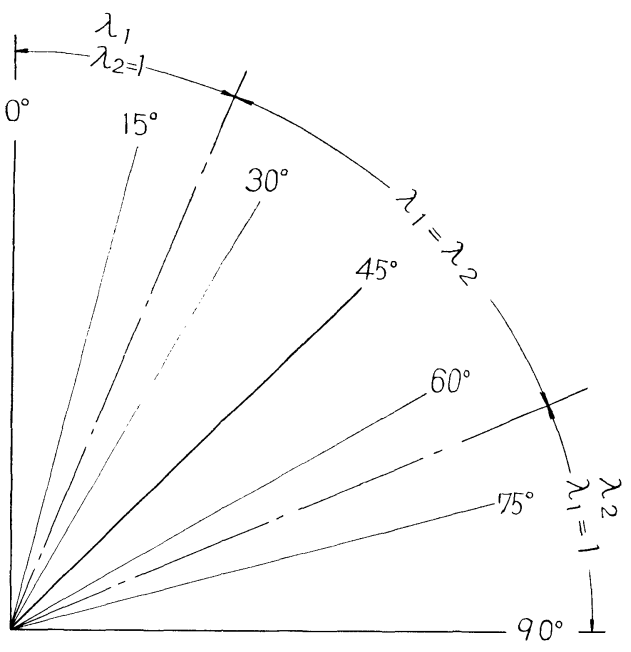

Fig. 21 Deformation of the fabric is approximated being equal to homogeneous-biaxial extension for $\Theta=$ $45^{\circ} \pm 22.5^{\circ}$, strip-baxial deformation along warp for $0^{\circ}<\Theta<22.5^{\circ}$ and strip-biaxial deformation along weft for $67.5^{\circ}<\Theta<90^{\circ}$

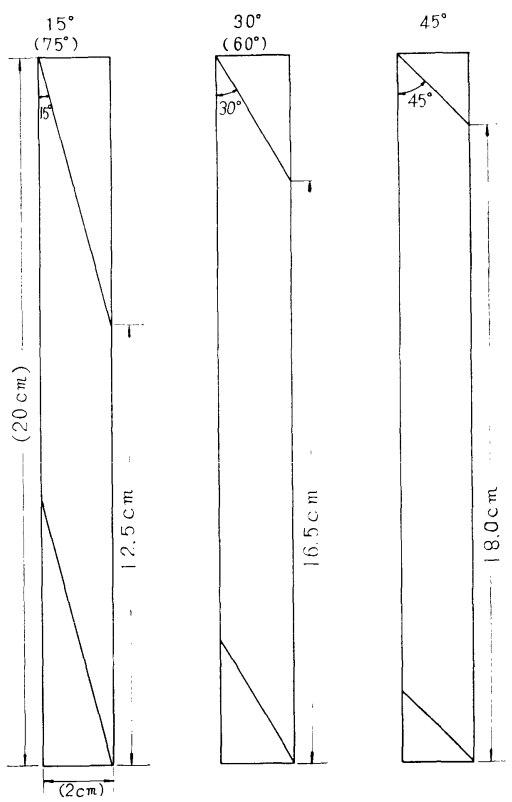

Fig. 22 Effective length of the specimen

extension rather than homogeneous one. A rule regarding to the se'ection of the values of $k_{1}$ and $k_{2}$ is shown in Fig.21. And this set of $k$ values can be obtained by biaxial deformation theory ${ }^{[3]}$. Substituting these values into eq.(49), $\Psi_{1}$ and $\Psi_{2}$ are obtained, and $f_{\Theta}$ is calculated as function of $\lambda$. An example of the calculated results is shown with experimental data in Fig.20. When $\Theta$ is less than $15^{\circ}$ or larger than $75^{\circ}$, the agreement is much better than the previous method.

From this facts, for the calculation of the unixial tensile property, it seems to ke necessary to select the use of the 


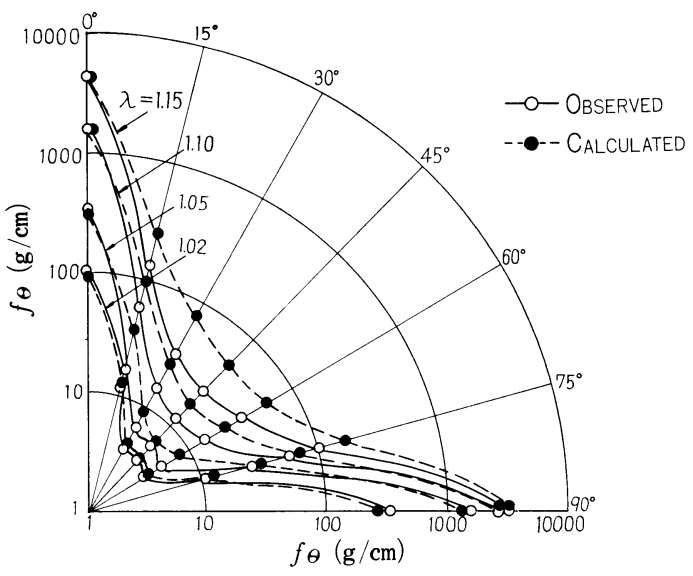

(a) uniaxial extension

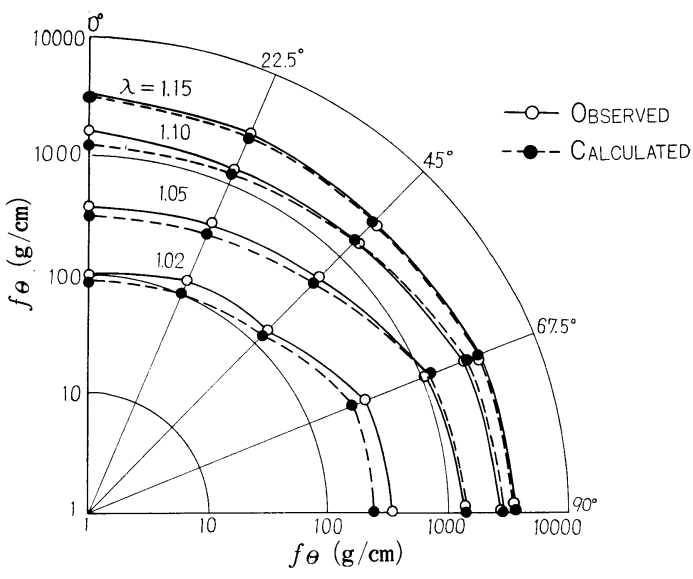

(b) strip-biaxial extension

Fig. 23 Anisotropy in the tensile properties of No. 3 fabric woven from polyester spun yarn

values of $k_{1}$ and $k_{2}$ in accordance with $\Theta$.

From the view point of experiment, the uniaxial testing is very difficult. One of the reason is the bending of specimen shown in Fig. 19 and the problem to decide the effective length. Fig.22 shows the effective length taken in this experiment.

4-2. Comparison of Anisotropic Properties of Biaxial and Uniaxial Extension.

In general, extreme anisotropy is observed in the property of uniaxial extension but the anisotropy becomes very small in biaxial extension as shown in Fig.23.

4-3. Relation between the Anisotropy in Tensile Properties and the Shear Deformation Properties

S.M. Spivak ${ }^{[14]}$ et al. tried to predict shear properties of the fabric in simple shear ${ }^{[15]}$ by the measurement of the tensile property along $\Theta=45^{\circ}$, and concluded being impossible to do it.

From this theory, this result is easily predicted because the uniaxial tensile properties is very hard to solve without some approximation.

However, the theory presented here predicts that the torque constants which caracterize the shear property can be calculated from the anisotropic properties of biaxial extension, that is, $F_{\theta}$ and $F_{\theta L}$ measured by the biaxial experiment of any $\Theta$ and $F_{1}$ and $F_{2}$ measured by the experiment of $\Theta=0^{\circ}$ are substituted into eqs.(13) and (14) to obtain $F_{t r 1}$ and $F_{t r 2}$. The value of $F_{c}$ is also calculated by the biaxial deformation theory ${ }^{\lfloor 3]}$.

From eqs.(16), (17) and (18) torque constants, $T_{r o}, C_{1}$, $C_{2}, C_{3}$ and $C_{4}$ can be determined.

These constants characterize the shear property of plain woven fabrics. ${ }^{[3,9,10]}$

\section{Conclusion}

The anisotropy of the biaxial tensile property including the uniaxial property has been solved theoretically in this paper by applying the shear deformation theory of plain woven fabrics already developed.

In this theory, the fabric structure and the following properties of yarns such that tensile property, compressible property against side pressure, and torque property at crossing point of warp and weft, are given.

On the other hand, the measurement of the anisotropy has been carried out and the results have been compared with theoretically calculated results and experimental ones.

This theory is constructed by very small approximations except the case of uniaxial deformation.

The agreement between theory and experiment is very good all over the full region of the stretching for any value of $\Theta$. But uniaxial tensile deformation is a special case of the biaxial tensile deformation, and some approximation is required in this case. And from this, some disagreement between them is observed in the stretching region of relatively high stretch ratio.

As is clear from the analysis presented here, the infinitesimal theory of anisotropic-continuous materials can not be applied to analyze the stress-strain properties of fabrics.

But this theory presented here can be used for investigations and design of fabrics instead of those conventional analyses.

In conclusion, we thank the memters of the Fiber Assembly Study Group of Dept. of Polymer Chemistry, Kyoto University, for their useful discussions and advices on this study.

This paper was presented at the 22nd Annual Meeting of the Textile Machinery Society of Japan (May 1969)

\section{Literature cited}

[1] M. Niwa, S. Kawabata, H. Kawai; J. Text. Mach. Soc. Japan, 19, T157 (1966); 20, T12, T258, T279 (1967) 
[2] S. Kawabata, M. Niwa, H. Kawai; Proc. to the 20th Annual Meeting of the Textile Machinery Society of Japan, 180 (1967)

[3] S. Kawabata, M. Niwa, H. Kawai; J. Text. Inst., in press.

[4] M. Niwa, S. Kawabata, H. Kawai; J. Text. Mach. Soc. Japan, 22, T85, T133 (1969)

[5] K. Weissenberg; J. Text. Inst., 40, T89 (1949)

[6] G.E. Chadwick, S.A. Shorter, K. Weissenterg; J. Text. Inst., 40, T111 (1963)

[7] W.F. Kilby; J. Text. Inst., 54, T 9 (1963)

[8] Fukaya; Aoyama-Gakuin Junior Women's College's Study Report, No.7 (1957)

[9] S. Kawabata, M. Niwa, H. Kawai; Proc. at the 21st
Annual Meeting of the Textile Machinery Society Japan, p.124 (1968)

[10] M. Niwa; Doctrorate Thesis (1968)

[11] K. Sakaguchi, S. Kawabata, H. Kawai, N. Hazama; Material, 17, 356 (1968)

[12] M. Niwa, S. Kawabata, H. Kawai; Proc. at the 21st Annual Meeting of the Textile Machinery Society of Japan, p.130 (1968)

[13] M. Niwa, S. Kawabata, H. Kawai, J. Fib. Sci. Tech. Japan, 26, 467, 507 (1970)

[14] S.M. Spivak, L.R.G. Treloar; Text. Res. J., 38, 963 (1968)

[15] S.M. Spivak; Text. Res. J., 36, 1056 (1966) 